\title{
Reconstruction of in-line holograms combining model fitting and image-based regularized inversion
}

\author{
Berdeu Anthony ${ }^{1}$, Flasseur Olivier ${ }^{1}$, Méès Loïc ${ }^{2}$, Denis Lö̈c ${ }^{1}$, Momey Fabien ${ }^{1}$, \\ Olivier Thomas $^{1}$, Grosjean Nathalie ${ }^{2}$, Fournier Corinne ${ }^{1, *}$ \\ ${ }^{1}$ Univ Lyon, UJM-Saint-Etienne, CNRS, Institut d Optique Graduate School, Laboratoire Hubert Curien UMR \\ 5516, F-42023, SAINT-ETIENNE, France \\ ${ }^{2}$ Laboratoire de Mécanique des Fluides et dAcoustique UMR CNRS 5509, École Centrale de Lyon, Université \\ Claude Bernard Lyon 1, INSA Lyon, 69134 Écully Cedex, France \\ * corinne.fournier@univ-st-etienne.fr
}

\begin{abstract}
We propose to reconstruct in-line holograms using a joint forward model on samples composed of a simple subpart estimated by model fitting and a complex shape subpart reconstructed using numerical (pixel-based) regularized inversion.

OCIS codes: (090.1995) Digital holography; (100.3010) Image reconstruction techniques; (100.3190) Inverse problems; (100.5070) Phase retrieval.
\end{abstract}

\section{Materials and methods}

To present the proposed method, we consider the case of evaporating droplets in the context of a fluid mechanics experiment. The method is primarily based on a split modelization of the object of interest in two subparts $u$ and $v$. The aim is to reconstruct $u$ and $v$ from their in-line intensity hologram $I_{d}$ under coherent illumination, composed of the interference of their respective contribution to the scattered wavefront $U_{d i f}$ on the sensor plane. From the linearity of the wave equation, this disturbance is the coherent summation of two waves $U_{d i f}^{p}$ and $U_{d i f}^{n p}$ which are the respective diffracted parts from $u$ and $v$, and for which two distinct diffraction models are available: an analytical - parametric - model for $U_{d i f}^{p}$ and a pixel-based - non-parametric - numerical model for $U_{d i f}^{n p}$.

Reconstructing $u$ and $v$ now consists in minimizing the following problem, assuming without loss of generality that $U_{\text {inc }}=1$ :

$$
(\hat{u}, \hat{v})=\underset{u, v}{\operatorname{argmin}}\left\|I_{d}-c \cdot\left|U_{i n c}+U_{d i f}^{p}(u)+U_{d i f}^{n p}(v)\right|^{2}\right\|_{W}^{2}
$$

where $c$ is a real-valued coefficient to account for the scaling of the acquisition dynamics and $W$ is a matrix of weighting coefficients to possibly account for the presence of defective pixels or the limited size of the sensor [1].

To solve the problem (1), an joint reconstruction scheme is implemented, alternating an optimization of the parametric model on $u$ and an optimization on the non-parametric model on $v$.

In the present case, the parametric object $u$ is the set of droplets. Assuming that a given droplet is a sphere of known refractive index, $U_{d i f}^{p}$ can be modeled via the Lorenz-Mie theory [2], which provides in the far field regime an analytical solution for the complex scalar field. The object $u$ is then described by a limited number of parameters: $u=\left\{u_{d}=\left(x_{d}, y_{d}, z_{d}, r_{d}\right)\right\}_{d \in \llbracket 1, N_{d} \rrbracket}$, respectively the 2D position of the droplets on the sensor plane, their distance to the sensor and their radius. For each particle $d$ in the field, the parameters $u_{d}$ are locally fitted with the Lorenz-Mie model [3].

The non-parametric object $v$ is the vapor wake generated by evaporation of the ether droplets. The unknown is modeled as a 2D map of its complex transmittance $t_{2 D}$. The diffracted wave $U_{d i f}^{n p}$ is produced by the difference of the transmittance to the unitary plane: $t_{2 D}-1$. After propagation from the object plane at a distance $z$, the resulting diffracted wave on the sensor plane is given by the convolution with the Rayleigh-Sommerfeld propagation kernel [4]. The transmittance map $t_{2 D}$ is reconstructed solving the problem (1) with respect to $v$ involving constraints and regularization terms favoring inter alia smoothness of the gradient of the image.

\section{Results}

Figure 1 presents the results of the proposed method on two evaporating droplets. The reconstruction distance $z$ is given by the fit of Mie model for the biggest droplet.

The reconstruction successfully retrieves the distances and sizes of the droplets as well was their evaporated vapor wake. In addition, it also estimates the background phase of the flow. Interestingly, it appears that the 
phase shift vanishes at the droplet position (see inset in figure 1.b-c). This supports the fact that the local phase information is refracted by the droplet and consequently lost.

By combining the two reconstructed subparts, once gets a global retrieved phase on the sensor plane (figure 1.g). Looking at figure 1.a, it appears that the final error in the residues is below $\pm 5 \%$ gray levels, with the very good agreement in the background, generally hard to reconstruct with standard methods because of the high contrast fringes produced by the droplets.

Figure 1.c and its inset show that the twin-image artifacts generally present in simple back-propagation are well cleaned by the regularization procedure.

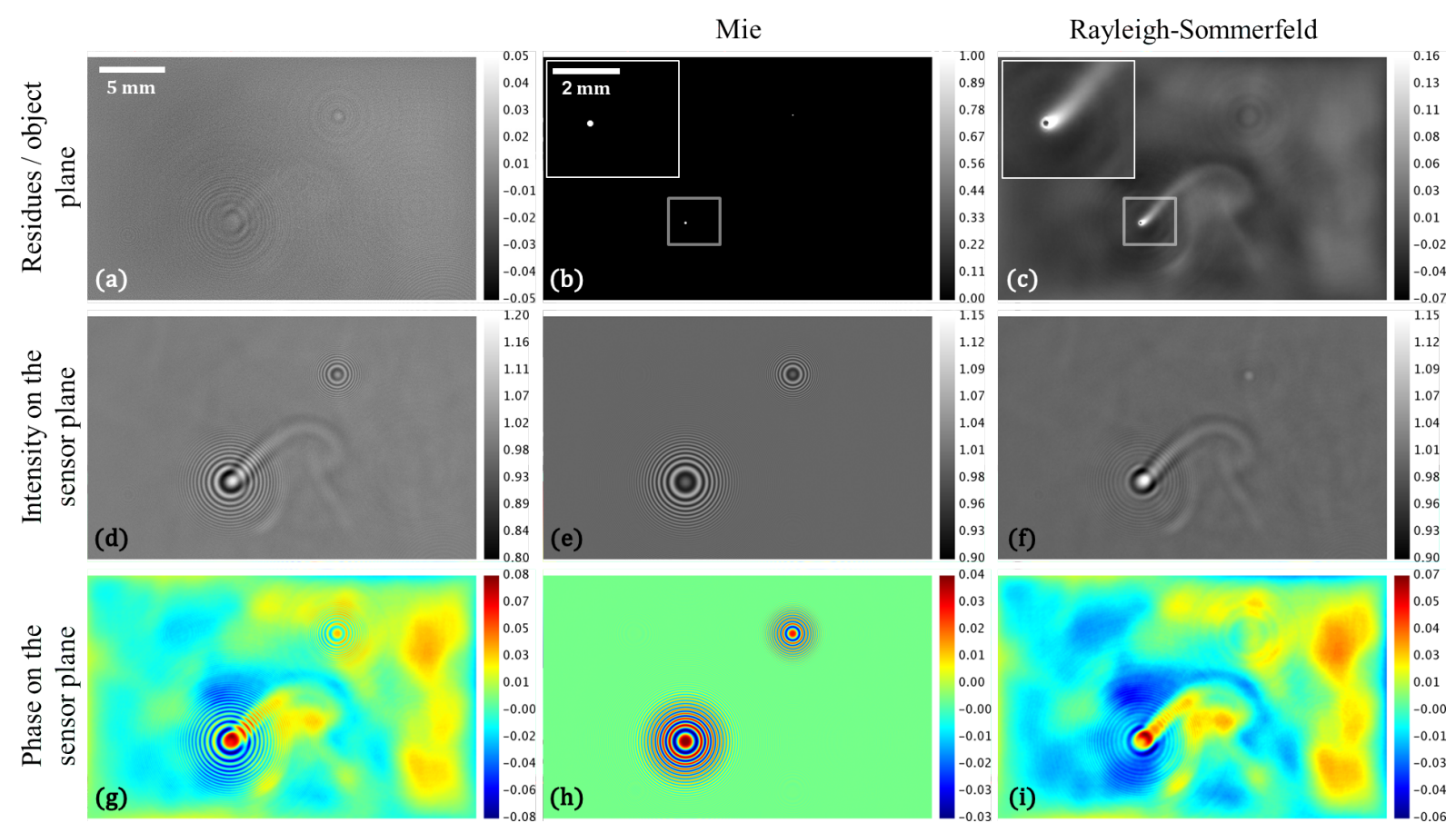

Fig. 1. Reconstruction of an in-line hologram of evaporating droplets. (a) Residues of the reconstruction.(b) Reconstruction absorption of the sample given by the Mie model. (c) Reconstructed phase delay induced by the vapor wake and the surrounding flow (in radians). (d) Initial raw hologram. (e,h) Intensity and phase (in radians) predicted by the Mie part of the model. (f,i) Intensity and phase (radian) predicted by the Rayleigh-Sommerfeld part of the model. (g) Retrieved phase (in radians) on the sensor plane.

\section{Conclusion}

In this work we provided the proof of concept that a combined parametric and non-parametric approach is feasible on single in-line holograms. The parametric part of the reconstruction effectively fits the high contrast fringes allowing the non-parametric part to retrieve the faintest parts of the object. These reconstructions are jointly performed, meaning that their interferences are rigorously modeled and taken into account, contrary to standard methods which work on subtracted intensities.

\section{References}

1. C. Fournier, F. Jolivet, L. Denis, N. Verrier, E. Thiebaut, C. Allier, and T. Fournel, "Pixel super-resolution in digital holography by regularized reconstruction," Appl. Opt. 56, 69-77 (2017).

2. L. Mees, N. Grosjean, D. Chareyron, J. L. Marie, M. Seifi, and C. Fournier, "Evaporating droplet hologram simulation for digital in-line holography setup with divergent beam," J. Opt. Soc. Am. Opt. image science, vision 30, 2021-2028 (2013).

3. F. Soulez, L. Denis, E. Thiebaut, C. Fournier, and C. Goepfert, "Inverse problem approach in particle digital holography: out-of-field particle detection made possible,” J. Opt. Soc. Am. A, Opt. image science, vision 24, 3708-3716 (2007).

4. J. W. Goodman, Introduction to Fourier optics (Roberts, Englewood (Colorado), 2005). 